\title{
HPV infection and P16 expression in oral and oropharyngeal cancer in Kazakhstan
}

\author{
Dauren Adilbay ${ }^{1 *} \mathbb{D}$, Galim Adilbayev², Gulzhan Kidirbayeva², Viktoria Shipilova², Zhanat Sadyk², \\ Gulsum Koyanbekova ${ }^{3}$, Ekaterina Sokolenko ${ }^{3}$ and Jan Klozar ${ }^{4}$
}

\begin{abstract}
Background: Human papillomavirus (HPV) is an important etiologic factor in different cancers of anogenital region and also in a fraction of head and neck cancers (HNC) particularly oropharyngeal tumors. The HPV16 genotype associated with the majority of HPV-related head and neck carcinomas. Th incidence of oropharyngeal cancer is increasing in many countries, and the rate of HPV positive tumors is about 70\% in Europe and North America. Little known about the prevalence of HPV in HNC in Central Asia.

Methods: It's a prospective analysis of patients with verified oral or oropharyngeal cancer. Sociodemographic and clinical data obtained on admission to treatment. The diagnosis of HPV positivity assessed by both the P16 expression on immunohistochemistry $(\mathrm{HC})$ and polymerase chain reaction (PCR)with HPV DNA detection and HR HPV type determination.

Results: Seventy six patients with oral and oropharyngeal cancer tested for HPV. Forteen cases were positive for HPV by PCR and 15 cases by P16 IHC. Of the 35 oropharyngeal tumors, nine were HPV DNA and P16 IHC positive, giving the rate of $25.7 \%$. Of the 41 oral tumors, five were HPV DNA and six p16 IHC positive, giving the rate of $12.2 \%$.

Conclusion: It is the first study mapping prevalence of HPV positivity in oral and oropharyngeal cancer in the Central Asian region. The rate of HPV positivity was higher in oropharyngeal than in oral cancer, the nonsmokers were significantly more frequent in the HPV positive group and HPV 16 was the most frequent type. However, the HPV positivity rates are lower than referred in the western world.
\end{abstract}

Keywords: HPV, Oral cavity, Oropharyngeal cancer, p16 IHC, Kazakhstan, This research was presented as an E-poster on IFOS 2017 ENT world congress (24-28 June) Paris, France. Abstract № HN-HPV-20.

\section{Background}

Oral cavity and oropharynx cancer is a major global health issue, they both constitute the majority of head and neck cancers. Globally 300,000 men and 130,000 women get diagnosed with these diseases annually, and the annual mortality is 160,000 and 68,000 respectively [1].

Main predisposing factors are smoking and alcohol consumption [2]. Despite the decrease in the incidence of tobacco-induced cancers in many countries, there is a rising incidence of oropharyngeal cancer worldwide. It is due to the increasing proportion of HPV-induced particularly oropharyngeal head and neck cancers $[3,4]$.

\footnotetext{
* Correspondence: dr.dauren@gmail.com

${ }^{1}$ Kazakh Institute of Oncology and Radiology, 91 Prospekt Abaya, Almaty, Kazakhstan050022

Full list of author information is available at the end of the article
}

It's known that HPV is an important etiologic factor in oropharyngeal cancer, yet, its role in other head and neck sites is unclear. There are more than a dozen of high-risk HPV types that can cause cancer. The 13 most frequent types of high-risk HPV (HR HPV) that are 16, $18,31,33,35,39,45,51,52,56,58,59$ and $68[5,6]$. Types 16 and 18 are the most frequently detected in cervical as well as in oropharyngeal cancers [5]. Most HR HPV positivity rate referred in up to $23 \%$ in oral cavity cancer and $24 \%$ in laryngeal cancer, but which is much less than in oropharyngeal cancer, where $50-60 \%$ of cases are HPV positive [6-8]. HPV-associated oropharyngeal cancer has unique morphologic features $[9,10]$. These tumors are non-keratinizing squamous cell carcinomas with basaloid appearance and hyper-expression of p16. In contrast, the HPV negative tumors are usually 
keratinizing and consist of polygonal cells [9]. Worldwide incidence of these cancers ranges from $14 \%$ to $85 \%$ depending on the region $[1,11,12]$. From a clinical point of view, the most important difference is the better prognosis of patients with HPV positive cancers. This data led to the change of TNM classification for HPV positive tumors and considerations about treatment deescalation [13, 14].

Kazakhstan is a multi-ethnic country located in Central Asia with a population of 17 million people. Its population is well known for high level of diversity, due to forced translocation of many nations during the Soviet period and a mixed structure of Kazakh nation [15]. The variety of population makes the possibility to get exciting results regarding the overall prevalence of HPV in the community, the distribution of HPV types and possibly also the relation to ethnicity. Up to date, there is only one published paper mapping the HPV prevalence in PAP smears of Kazakhstan women [16]. Each year more than 700 patients are diagnosed with oral cavity and oropharyngeal cancer in Kazakhstan [17, 18], and up to date, no studies were investigating HPV-associated head and neck cancer.

The role of HPV in cancerogenesis is now well documented, and the biological and clinical differences between HPV positive and negative tumors will probably very soon have consequences also in treatment approach [19]. From this point, it is essential to get the data of prevalence and differences in ethnic-geographic distribution in Kazakhstan. It will give the possibility to plan awareness campaigns and provide personalized treatment to patients in this country.

\section{Methods}

It is a prospective analysis of patients with verified oral or oropharyngeal cancer referred to a single tertiary institution (observational study). Sociodemographic and clinical data obtained through a questionnaire on admission to treatment. Approval for the study received from the local institutional ethics committee, and informed consent obtained from all study participants.

The study enrolled 76 patients with oral and oropharyngeal cancer treated between February 2015 and May 2017 in Kazakh Institute of Oncology and Radiology. All patients have squamous cell carcinoma of different grades. Out of 76 patients, 35 had oropharyngeal cancer and 41 oral cavity cancer.

Part of the fresh tumor tissue from a biopsy specimen taken for PCR analysis and p16 IHC staining performed on formalin fixed paraffin embedded slices.

\section{HPV-DNA PCR}

RIBO-sorb nucleic acid extraction kit was used to extract and purify DNA from cancer tissue. RIBO-sorb nucleic acid extraction kit is reagents kit for rapid and efficient manual extraction and purification of DNA from various biological materials.

AmpliSens HPV HCR genotype-FRT PCR kit is an in vitro nucleic acid amplification test for qualitative detection and differentiation of high risk (HR) human papillomaviruses (HPV) types 16, 18, 31, 33, 35, 39, 45, 51, 52, $56,58,59 \mathrm{DNA}$ in the clinical material by using realtime hybridization-fluorescence detection of amplified products [5].

The test based on simultaneous PCR (multiplex-PCR) and real-time detection of three HPV types and b-globin gene DNA, used as internal control, in one tube. The analysis of $12 \mathrm{HPV}$ types is carried out in four tubes. Each HPV type registered on its channel that allows not only to detect but also to differentiate the virus genotype. The DNA target selected as an endogenous internal control is a human genome fragment. AmpliSens HPV HCR genotype-FRT PCR kit uses "hot-start," which significantly reduces the frequency of nonspecifically primed reactions.

\section{p16 immunohistochemistry}

p16 IHC was carried out using the CINtec Histology kit (mtm Laboratories, AG, Germany) on a Ventana Benchmark Autostainer. p16 IHC was scored as positive if there was strong and diffuse nuclear and cytoplasmic staining present in greater than $70 \%$ of the malignant cells. All other staining patterns scored as negative. All samples were scored independently by two expert pathologists, and discordant cases were reviewed to come to a consensus score. Examinations were carried out at the Department of Pathology of Kazakh Institute of Oncology and Radiology.

\section{Results}

There were 76 patients with oral and oropharyngeal cancer enrolled in the study. Of them 35 had oropharyngeal and 41 oral cavity cancer, 50 were male and 26 female. Mean age was 57.2 years (standard deviation 11.45 years), with youngest and oldest participant were 26 and 79 respectively. Patients characteristics presented in Table 1.

Of the 76 patients, 14 cases were positive for HPV by PCR and 15 by P16 IHC. One patient had positive p16 IHC and negative PCR test. Of the 35 oropharyngeal tumors, nine were HPV DNA and p16 IHC positive, giving the rate of $25.7 \%$. Out of nine positive cases, four are tonsillar cancers and two base of tongue cancers, what corresponds with the distribution of the whole group of oropharyngeal tumors.

Of the 41 oral tumors, five were HPV DNA positive and six patients were p16 IHC positive, giving the rate of $12.2 \%$ (excluding the P16 only positive case). Three of 
Table 1 Patients characteristics

\begin{tabular}{lllllll}
\hline Primary Site & II stage & III stage & IV stage & Male & Female & Total \\
\hline Oropharyngeal cancer & $10(28.6 \%)$ & $19(54.3 \%)$ & $6(17.1 \%)$ & $25(71.4 \%)$ & $10(28.6 \%)$ & $35(46.1 \%)$ \\
Tonsillar & 5 & 10 & 3 & 13 & 5 & $18(51.4 \%)$ \\
Base of tongue & 3 & 3 & 0 & 4 & 2 & $3(17.1 \%)$ \\
Other & 2 & 6 & 3 & 8 & $11(31.4 \%)$ \\
Oral cavity cancer & $12(29.3 \%)$ & $25(61.0 \%)$ & $4(9.8 \%)$ & $25(61 \%)$ & $16(39 \%)$ & $41(53.9 \%)$ \\
Floor of mouth & 5 & 6 & 2 & 10 & 3 & $13(31.7 \%)$ \\
Tongue & 5 & 16 & 2 & 0 & 2 & $23(56.1 \%)$ \\
Buccal mucosa & 1 & 1 & 0 & 1 & $2(4.9 \%)$ \\
Alveolar ridge, retromolar trigone & 1 & 2 & 0 & & $3(7.3 \%)$ \\
\hline
\end{tabular}

them are tongue cancer, one floor of the mouth and one alveolar ridge tumors. All three tongue cancers were located closer to posterior third of the tongue and even with extension to the base of the tongue.

HPV 16 was the most prevalent type in both the oropharyngeal and oral cavity cases, other types found were 18 and 56, one case each (Table 2).

Smoking was less prevalent in the group of HPV positive patients, two had a history of smoking out of 14, comparing 40 out of 62 in the HPV negative group of patients. The difference is statistically significant with $p$ $=0.0018$ (Chi-squared test).

In the oropharyngeal cancer group, 25 patients had advanced tumors stage III and IV. Patients with oropharyngeal cancer received concurrent chemoradiotherapy (CRT) according to the local standards. The local CRT standard includes delivering IMRT radiotherapy to tumor and neck with a concurrent infusion of platinumbased agent weekly or every three weeks.

In the group of oral cancers, 29 out of 41 had advanced oral cancer stage III and IV. They were treated mostly by surgery and adjuvant RT or CRT for non-operable cases. Adjuvant CRT in the oral cavity cancer group is used only for cases with adverse features on permanent pathology.

\section{Discussion}

Cancers of the head and neck is a heterogeneous group of tumors with various anatomic sites with different etiologic factors. Tobacco exposure (either via active or passive smoking as well as consumption of smokeless tobacco) is the leading risk factor for head and neck cancers [2]. But in many developed nations with decreasing tobacco usage and consequently decreasing the incidence of tobacco induced cancers the incidence of oropharyngeal cancer is rising. This development attributed to the spread of $\mathrm{HR}$ HPV infection [1, 20, 21]. The data gathered in different multinational trials confirms the need for evaluation of HPV prevalence by region and country, as there are important differences between patient groups from Western Europe, Eastern Europe, and Asia [3]. As mentioned in many systematic reviews and meta-analysis one of the main limitations for comparing different studies was the lack of uniformity of the tests used [8]. In this study, we attempt to overcome this limitation by the use of commercial kits for both the p16 ICH and HPV DNA detection and type determination.

Kazakhstan is a partly Eastern European, partially Asian state with a mixed population. The primary risk factor for the local population is the smoking, as there is no or very low level of smokeless tobacco consumption prevalent in other Asian countries. The incidence of HNC is low in Kazakhstan when compared with, e.g., Europe. However, we can follow similar trends. There is rising agestandardized incidence rate of oral and oropharyngeal cancer from 3.8 in 2004 to 4.7 in 2015 per 100,000. In the same time incidence of laryngeal cancer mostly attributed to smoking has decreased from 2.6 in 2004 to 2.3 in 2015 [22]. Similarly, as in other countries this trend can be attributed to rising number of HPV induced oropharyngeal cancer, but further studies needed to establish the liaison, as some trials show the stable percentage of HPV positive oropharyngeal cancers over a decade [23]. In our study, most of the positive cases are from the oropharynx. Even several cases from oral cavity are from sites anatomically closer to

Table 2 HPV-induced cancer prevalence and types detected

\begin{tabular}{|c|c|c|c|c|c|c|c|c|c|}
\hline \multirow[t]{2}{*}{ Primary Site } & \multirow[t]{2}{*}{$\mathrm{P} 16+(\mathrm{IHC})$} & \multicolumn{3}{|l|}{$\mathrm{HPV}+$} & \multirow[t]{2}{*}{ HPV - } & \multirow[t]{2}{*}{ HPV+ prevalence } & \multicolumn{2}{|c|}{ 95\% Cl (Wilson) } & \multirow[t]{2}{*}{ Total } \\
\hline & & HPV16 & HPV18 & HPV56 & & & Lower & Upper & \\
\hline Oropharyngeal cancer & 9 & 8 & 1 & 0 & 26 & 0.257 & 0.141 & 0.421 & 35 \\
\hline Oral cavity cancer & 6 & 4 & 0 & 1 & 36 & 0.122 & 0.053 & 0.255 & 41 \\
\hline All sites & 15 & 12 & 1 & 1 & 62 & 0.184 & 0.113 & 0.286 & 76 \\
\hline
\end{tabular}


the oropharynx, like the posterior third of the tongue or mandibular alveolar ridge. This site dependence is the same as described in many trials performed worldwide $[1,3]$.

\section{Conclusion}

In summary in this first study in the Central Asian region the prevalence of HPV induced oral and oropharyngeal cancer is lower than in Europe and North America, but higher than in other Asian countries [3, 21]. In concordance with European and American results, the rate of HPV positivity was higher in oropharyngeal than in oral cancer, the nonsmokers were significantly more frequent in the HPV positive group and HPV 16 was by far the most frequent type. Further studies are needed to establish a better understanding of HPV prevalence and time trends in head and neck cancers in Kazakhstan. This trial provides the first valuable information for public policy and healthcare workers to plan screening, awareness campaigns, vaccination and in the future also the treatment of these tumors.

\section{Acknowledgements}

Ablay Kerimkulov, Svetlana Kauss and other lab workers that were involved in $\mathrm{IHC}$ and PCR are much acknowledged. The publication is supported by the educational grant from Pfizer.

\section{Funding}

Research funded by the Kazakhstan Ministry of Science and Education grant № 1080/ГФ4 from 12.02.2015.

\section{Availability of data and materials}

A majority of data generated or analyzed during this study included in this published article. The datasets used and/or analyzed during the current study available from the corresponding author on reasonable request.

\section{Authors' contributions}

All authors collaborated in the study design; DA, GA, VS, ZS participated in diagnosing and managing these patients; GK and ES performed PCR and ICH of these patients; DA, ZS, GK, VS extracted and analyzed the clinical data; DA ZS elucidated the data and carried out statistical analysis; DA prepared the first manuscript draft; JK modified the manuscript subsequently; all authors read and approved the final manuscript.

\section{Ethics approval and consent to participate}

Approval for the study obtained from the local institutional ethics committee and informed consent obtained from all study participants.

\section{Consent for publication}

Not applicable.

\section{Competing interests}

The authors declare that they have no competing financial or non-financial interests.

\section{Publisher's Note}

Springer Nature remains neutral with regard to jurisdictional claims in published maps and institutional affiliations.

\section{Author details}

${ }^{1}$ Kazakh Institute of Oncology and Radiology, 91 Prospekt Abaya, Almaty, Kazakhstan050022. ${ }^{2}$ Head and neck oncology center, Kazakh Institute of Oncology and Radiology, Almaty, Kazakhstan. ${ }^{3}$ Pathology and molecular biology department, Kazakh Institute of Oncology and Radiology, Almaty, Kazakhstan. ${ }^{4}$ Charles University in Prague, University Hospital Motol, Prague, Czech Republic.
Received: 22 September 2017 Accepted: 5 January 2018

Published online: 12 January 2018

\section{References}

1. Chaturvedi AK, et al. Worldwide trends in incidence rates for oral cavity and oropharyngeal cancers. J Clin Oncol. 2013;31(36):4550-9.

2. Ansary-Moghaddam A, et al. Risk of upper Aerodigestive tract cancer associated with smoking with and without concurrent alcohol consumption. Mt Sinai J Med. 2009;76(4):392-403.

3. Mehanna $H$, et al. Geographic variation in human papillomavirus-related oropharyngeal cancer: data from 4 multinational randomized trials. Head and Neck-Journal for the Sciences and Specialties of the Head and Neck. 2016;38:E1863-9.

4. Gooi Z, Chan JYK, Fakhry C. The epidemiology of the human papillomavirus related to oropharyngeal head and neck cancer. Laryngoscope. 2016;126(4):894-900.

5. Haghshenas $M$, et al. Prevalence and type distribution of high-risk human papillomavirus in patients with cervical cancer: a population-based study. Infectious Agents and Cancer. 2013;8:6.

6. Kreimer AR, et al. Human papillomavirus types in head and neck squamous cell carcinomas worldwide: a systematic review. Cancer Epidemiol Biomark Prev. 2005;14(2):467-75.

7. Marur S, et al. HPV-associated head and neck cancer: a virus-related cancer epidemic. Lancet Oncol. 2010;11(8):781-9.

8. Mehanna $\mathrm{H}$, et al. Prevalence of human papillomavirus in oropharyngea and nonoropharyngeal head and neck cancersystematic review and metaanalysis of trends by time and region. Head and Neck-Journal for the Sciences and Specialties of the Head and Neck. 2013;35(5):747-55.

9. Cai CY, et al. Keratinizing-type squamous cell carcinoma of the oropharynx p16 overexpression is associated with positive high-risk HPV status and improved survival. Am J Surg Pathol. 2014;38(6):809-15.

10. El-Mofty SK. Human papillomavirus-related head and neck squamous cell carcinoma variants. Semin Diagn Pathol. 2015;32(1):23-31.

11. Herrero R, et al. Human papillomavirus and oral cancer: the international agency for research on cancer multicenter study. J Natl Cancer Inst. 2003; 95(23):1772-83.

12. St Guily $J$, et al. Human papillomavirus genotype distribution in oropharynx and oral cavity cancer in France-the EDiTH VI study. J Clin Virol. 2011;51(2):100-4.

13. Klozar J, Tachezy R. What are the implications of human papillomavirus status in oropharyngeal tumors for clinical practice? Current Opinion in Otolaryngology \& Head and Neck Surgery. 2014;22(2):90-4.

14. Doescher, J., J.A. Veit, and T.K. Hoffmann, The 8th edition of the AJCC Cancer Staging Manual. Updates in otorhinolaryngology, head and neck surgery. Hno, 2017. 65(12): p. 956-+.

15. Altynay, K., et al., Kazakhstan's experience in the enhancement of the intercultural dialogue in a multicultural society. 3rd Cyprus International Conference on Educational Research (Cy-Icer 2014), 2014. 143: p. 912-915.

16. Niyazmetova $\mathrm{L}$, et al. Application of molecular genotyping to determine prevalence of HPV strains in pap smears of Kazakhstan women. Int J Infect Dis. 2017:54:85-8.

17. Adilbay DG, Chichua NA, Adilbayev GB. CONCURRENT CHEMORADIOTHERAPY FOR LOCALLY ADVANCED ORAL CAVITY CANCER (T3-4N0-2M0). Journal of N. N. Blokhin Russian Cancer Research Center RAMS. 2009;20(3):21-7.

18. Adilbayev $\mathrm{G}$, et al. Immediate results HPV-induced cancer of the mouth and oropharynx in Kazakhstan. Ann Oncol. 2016:27:1.

19. Vojtechova Z, et al. Analysis of the integration of human papillomaviruses in head and neck tumours in relation to patients' prognosis. Int J Cancer. 2016, 138(2):386-95

20. Betiol JC, et al. Prevalence of human papillomavirus types and variants and p16(INK4a) expression in head and neck squamous cells carcinomas in Sao Paulo, Brazil. Infectious Agents and Cancer. 2016;11:7.

21. Liederbach $E$, et al. The national landscape of human papillomavirusassociated oropharynx squamous cell carcinoma. Int J Cancer. 2017; 140(3):504-12.

22. Dilyara K, et al. Pokazateli onkologicheskoi sluzhbi Respubliki Kazakhstan za 2015 god. 2016. KazNII oncologii i radiologii: Almaty.

23. Schache AG, et al. HPV-related oropharynx cancer in the United Kingdom: an evolution in the understanding of disease etiology. Cancer Res. 2016; 76(22):6598-606. 